\title{
Comparison Study on Different Quantification Methods of Diffuse Myocardial Fibrosis of Dilated Cardiomyopathy Using Myocardial T1 Value*
}

\author{
Takeshi Hayashida, Eijun Sueyoshi", Hiroki Nagayama, Ichiro Sakamoto, Masataka Uetani \\ Department of Radiology, Nagasaki University School of Medicine, Nagasaki, Japan \\ Email: "sueyo@nagasaki-u.ac.jp
}

Received June 26, 2013; revised July 26, 2013; accepted August 3, 2013

Copyright (C) 2013 Takeshi Hayashida et al. This is an open access article distributed under the Creative Commons Attribution License, which permits unrestricted use, distribution, and reproduction in any medium, provided the original work is properly cited.

\begin{abstract}
Purpose: The purpose is to compare several quantification methods and clarify which quantification method is reliable to estimate diffuse myocardial fibrosis with cardiac MRI in patients with dilated cardiomyopathy (DCM) using myocardial T1 value. Methods and Results: Delayed enhancement imaging was performed in 52 patients with DCM and 10 control subjects to identify fibrosis using an inversion time scout sequence. The mean contrast-enhanced myocardial (M) T1 values of the pre and post contrast-enhanced myocardial and left ventricular lumen (L) of control and dilated cardiomyopathy cases were compared. The calculated post M T1 value, pre M T1 value-post M T1 value, and (pre M TI value-post $\mathrm{M} \mathrm{T1}$ value)/(pre L T1 value-post L T1 value) were significantly different between the patient group and the control group ( $344.5 \pm 31.6$ vs. $390.4 \pm 19.3 \mathrm{msec}, 239.9 \pm 64.2 \mathrm{msec}$ vs. $134.0 \pm 28.9 \mathrm{msec}$, and $0.50 \pm 0.11$ vs. $0.30 \pm$ 0.60 , respectively). (Pre M T1 value-post M T1 value)/(pre L T1 value-post L T1 value) was significantly the most related to the left ventricular ejection fraction $(r=0.66, p<0.0001)$. Conclusion: (Pre M T1 value-post M T1 value)/(pre $\mathrm{L}$ T1 value-post L T1 value) was the most reliable quantification method to estimate the severity of DCM.
\end{abstract}

Keywords: Inversion Time; Quantification Methods; Myocardial Fibrosis; Dilated Cardiomyopathy

\section{Introduction}

Recently, the importance of myocardial fibrosis in the development and progression of systolic and diastolic cardiac failure has been highlighted in cardiomyopathy [1-3]. Central to the diagnostic utility of cardiovascular magnetic resonance is its almost unique capacity to reveal myocardial fibrosis through the use of delayed gadolinium enhancement imaging [4].

In patients with cardiac infarction, infarcted regions in the myocardium, having undergone scar formation with collagen deposition, have a much slower washout rate of gadolinium-based contrast material than healthy myocardium, leading to markedly increased signal intensity on T1-weighted imaging [5]. On the other hand, because $60 \%$ of dilated cardiomyopathy (DCM) patients have diffuse myocardial fibrosis, the technique of delayed contrast enhancement often shows no regional scarring [5]. Previous study suggested that the mean contrastenhanced myocardial inversion time at the null point (T1

\footnotetext{
${ }^{*}$ Conflict of interest: This work was supported by JSPS KAKENHI Grant Number 24591776.

${ }^{\#}$ Corresponding author.
}

value) was significantly shorter in a patient group than in a control group [5]. T1 value can be easily obtained, but this technique does not consider the influence of the contrast medium in the blood pool, and thus may not evaluate the enhancement of myocardium precisely.

The purpose of this study was to compare several quantification methods and clarify which quantification method is reliable to evaluate diffuse myocardial fibrosis of DCM using T1 value.

\section{Materials and Methods}

\subsection{Patients}

Fifty-two patients (33 men, 19 women; mean age \pm SD, $53.3 \pm 13.6 \mathrm{y}$; age range, $18-77 \mathrm{y}$ ) with DCM and 10 control subjects ( 5 men, 5 women; mean age \pm SD, 54.8 $\pm 12.4 \mathrm{y}$; age range, $55-74 \mathrm{y})$ underwent MRI at our institution between June 2008 and July 2011. There was no statistically significant difference in mean age $(P=$ $0.763)$ between the two groups. The diagnosis of nonischemic DCM was made according to World Health Organization and International Society and Federation of 
Cardiology criteria [6]. None of the subjects had clinical symptoms or signs of ongoing myocarditis. By the use of coronary angiography, cases of significant coronary artery disease $(>50 \%$ diameter luminal stenosis in any coronary artery) were excluded from this study. Other exclusion criteria were the presence of any contraindications for cardiac MRI, significant valvular disease, or any evidence of infiltrative heart disease by clinical symptoms or signs and sonographic findings. In addition, all patients with DCM underwent myocardial biopsy. The specimens showed disarray, with varying degrees of interstitial fibrosis of the myocardium, which were consistent with DCM.

On the other hand, all control subjects $(n=10)$ underwent cardiac MRI because of arrhythmia; however, no subjects had abnormalities according to coronary angiography, laboratory testing, echocardiography, nuclear medicine scan, or myocardial biopsy, which were performed to rule out various myocardial diseases.

All subjects gave written informed consent, and the protocol was approved by a medical ethics committee.

\subsection{Cardiac MRI}

All subjects were studied in the supine position using a 1.5-T cardiac MRI system (Avanto, Siemens Healthcare) with an eight-channel body coil. The data acquisition was blinded to diagnosis. The cardiac MRI study included cine steady-state free-precision imaging (TR/TE, 3.4/1.2; in-plane spatial resolution, $1.6 \times 2 \mathrm{~mm}$ ) of left ventricular function. Cine images were acquired with ECG gating and breath-holding. Cine images were obtained in $8-14$ short axis $(8-\mathrm{mm}$ thickness with 0 -mm spacing) from the base to the apex of the left ventricle. Vertical long axis images were also obtained. In all subjects $(n=62)$, inversion time scout images were obtained before enhancement and 15 minutes from the beginning of bolus injection of gadopentetate dimeglumine $(0.2 \mathrm{mmol} / \mathrm{kg}$; Magnevist, Bayer Schering Pharma) to identify regional fibrosis using a $2 \mathrm{D}$ inversion recovery gradient-echo technique (inversion time scout sequence: TR/TE, 20.8/1.3; flip angle, $25^{\circ}$; acquisition matrix, $192 \times 78$; field of view, $34 \times 27 \mathrm{~cm}$; slice thickness, $8 \mathrm{~mm}$; inversion time, individually determined to null the myocardial and left ventricular lumen signal). Forty-two images per single midventricular slice were acquired at various inversion times during breath-holding as long as possible (Figures 1(a) and (b)). This single midventricular slice was selected on the basis of long axis scout images of the left ventricle be- fore inversion time scout images were obtained. These inversion time scout images were then processed with a curve-fitting technique to generate T1 maps (Figures 1(c) and (d)). After the inversion time scout sequence was performed, ordinary delayed enhanced images were obtained.

\subsection{Data Analysis}

All MRI post-processing was performed by a single observer with more than 10 years of experience in cardiac MRI. The left ventricular ejection fraction was derived from cine images using a workstation for analysis (Syngo, Siemens Healthcare). A T1 mapping sequence was used to calculate the pre and post contrast-enhanced myocardial and left ventricular lumen T1 times of a single midventricular slice as an index of diffuse fibrosis. After image acquisition, short-axis images of varying inversion times were transferred to a workstation for analysis (Syngo, Siemens Healthcare). For each image, a region of interest was drawn in the left ventricular lumen and around the entire myocardium to calculate the pre and post contrast-enhanced myocardial and left ventricular lumen TI0 values for each subject (Figures 1(a) and (b)). This allowed us to analyze regions of interest to find the average $\mathrm{T} 1$ time for that area, as well as a pixel-by-pixel determination of $\mathrm{T} 1$, by fitting data acquired at various preparation times to the exponential curve.

We then determined pre and post contrast-enhanced myocardial (M) and left ventricular lumen (L) T1 values (pre contrast-enhanced myocardial T1 value; pre M T1 value, pre contrast-enhanced left ventricular lumen $\mathrm{T} 1$ value; pre $\mathrm{L} \mathrm{T} 1$ value, post contrast-enhanced myocardial $\mathrm{T} 1$ value; post $\mathrm{M} \mathrm{T1}$ value, post contrast-enhanced left ventricular lumen $\mathrm{T} 1$ value; post $\mathrm{L}$ T1 value) $[1,7,8]$ (Figures 1(c) and (d)). We compared the several quantification methods as follows: 1) post M T1 value, 2) pre $M$ T1 value-post $M$ T1 value, 3) post $M$ T1 value/post $L$ $\mathrm{T} 1$ value, and 4) (pre M T1 value-post M T1 value)/(pre $\mathrm{L}$ T1 value-post $\mathrm{L}$ T1 value).

\subsection{Statistical Analysis}

All values are expressed as the mean $\pm \mathrm{SD}$. Statistical analysis was performed on clinical and morphologic variables with the paired Student's $t$ test and MannWhitney $U$ test for continuous variables. Pearson's correlation coefficients were used to examine the correlation of left ventricular ejection fraction with all TI0 values. Correlation coefficient values of $0.4-1.0$ were considered to indicate a correlation [9]. In all tests, $p<0.05$ was considered significant (SPSS, release 11.5; SPSS).

\section{Results}

The results are summarized in Table 1. In patients in the DCM group, the mean pre M T1 value, post M T1 value, pre $\mathrm{L} T 1$ value, and post $\mathrm{L} \mathrm{T} 1$ value were $581.3 \pm 58.3$ $\mathrm{msec}, 344.5 \pm 31.6 \mathrm{msec}, 741.2 \pm 92.5 \mathrm{msec}$, and $257.5 \pm$ $46.1 \mathrm{msec}$, respectively. In the control group, the mean pre M T1 value, post M T1 value, pre L T1 value, and post L T1 value were $519.7 \pm 30.4 \mathrm{msec}, 390.4 \pm 19.3$ msec, $731.4 \pm 64.4 \mathrm{msec}$, and $299.3 \pm 35.5 \mathrm{msec}$, 


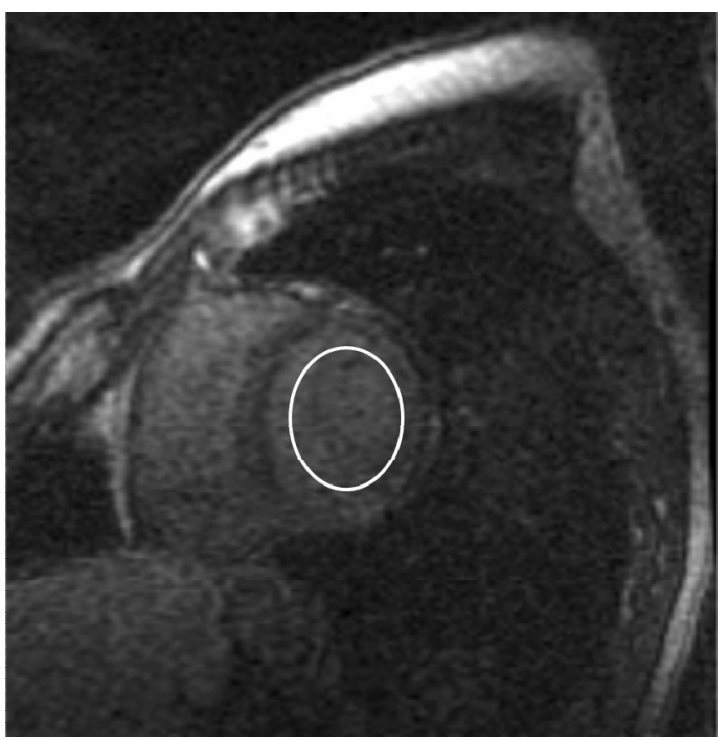

(a)

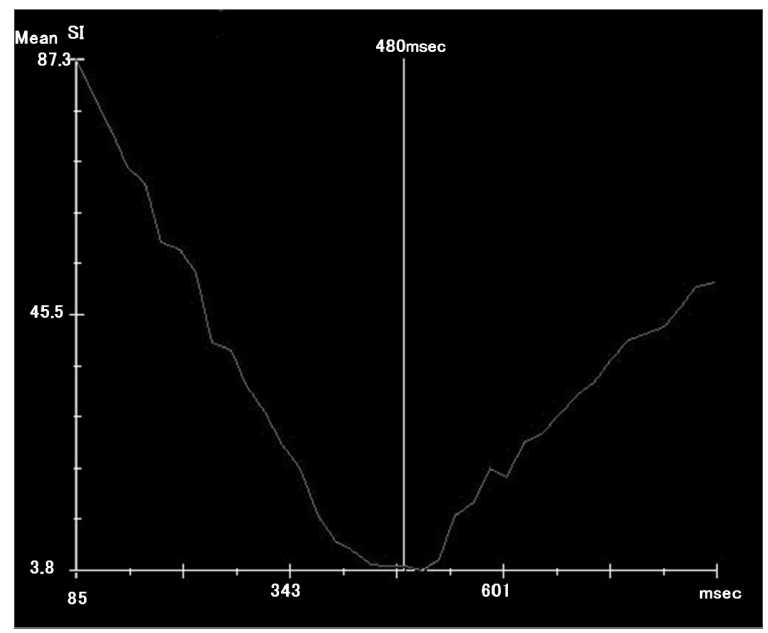

(c)

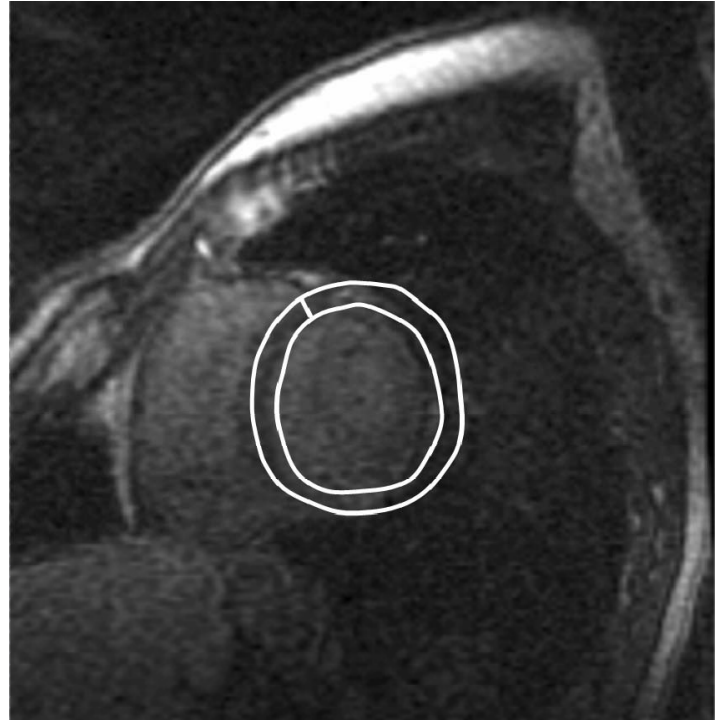

(b)

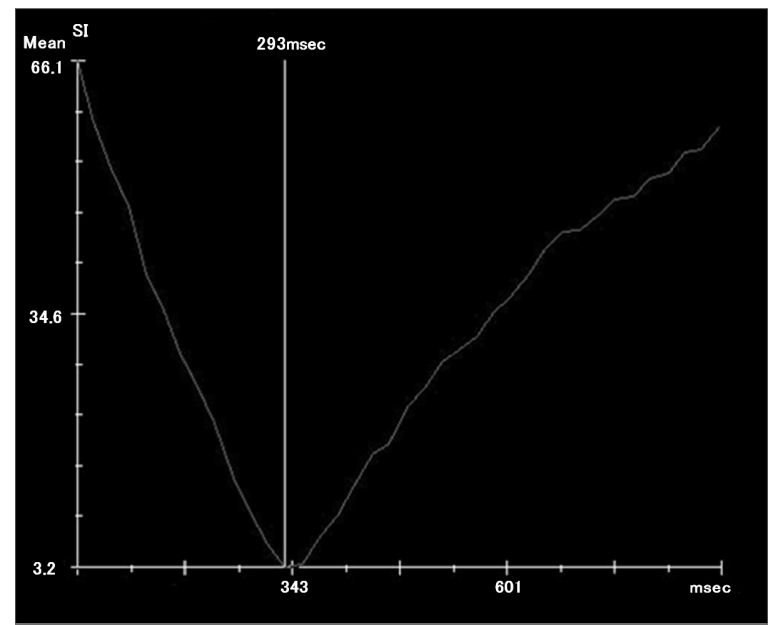

(d)

Figure 1. A 63-year-old woman with dilated cardiomyopathy. (a) Epicardial and endocardial borders were manually traced, and a region of interest was placed in a single midventricular slice to obtain signal intensity of left ventricular myocardium. (b) Region of interest was manually placed in the left ventricular lumen in a single midventricular slice to obtain signal intensity of left ventricular myocardium. (c) We analyzed regions of interest to find the average $\mathrm{T} 1$ for that area by fitting data acquired at various preparation times to an exponential curve. We determined pre contrast-enhanced myocardial $T 1$ value (vertical line). SI = signal intensity. (d) We analyzed regions of interest to find the average T1 for that area by fitting data acquired at various preparation times to an exponential curve. We determined post contrast-enhanced myocardial T1 value (vertical line). SI = signal intensity.

respectively (Table 2). There were statistically significant differences between the patient and control groups in pre $\mathrm{M}$ T1 values, post M T1 values, and post L T1 values ( $P=0.0052, P<0.0001, P=0.002$, respectively). However, there was no significant difference between the patient and control groups in pre L T1 value $(\mathrm{P}=0.559)$.

In patients in the DCM group, post M T1 value, pre $\mathrm{M}$ T1 value-post $M$ T1 value, post $M$ T1 value/post L T1 value, and (pre M T1 value-post M T1 value)/(pre L T1 value-post L T1 value) were $344.5 \pm 31.6 \mathrm{msec}, 239.9 \pm$ $64.2 \mathrm{msec}, 1.37 \pm 0.21$, and $0.50 \pm 0.11$, respectively. In the control group, post M T1 value, pre M T1 value-post M T1 value, post M T1 value/post T1 value, and (pre M T1 value-post $M$ T1 value)/(pre L T1 value-post L T1 value) were $390.4 \pm 19.3 \mathrm{msec}, 134.0 \pm 28.9 \mathrm{msec}, 1.31$ \pm 0.13 , and $0.30 \pm 0.60$, respectively (Table 3). There were statistically significant differences between the patient and control groups in post $\mathrm{M} \mathrm{T1}$ value, pre $\mathrm{M}$ T1 value-post $M$ T1 value, and (pre M T1 value-post M T1 value)/(pre L T1 value-post L T1 value) $(P<0.0001, P<$ $0.0001, P<0.0001$, respectively). However, there was no significant difference between the patient and control 
Table 1. Summary of results in patient and control groups.

\begin{tabular}{lccc}
\hline \multicolumn{1}{c}{ Characteristic } & $\begin{array}{c}\text { Control Subjects } \\
(\mathrm{n}=10)\end{array}$ & $\begin{array}{c}\text { Patients } \\
(\mathrm{n}=52)\end{array}$ & $P$ \\
\hline Age(Y) & $54.8 \pm 12.4$ & $53.3 \pm 13.6$ & NS \\
Sex(no.) & & 33 & NS \\
Male & 5 & 19 & \\
Female & 5 & $37.2 \pm 14.1$ & $<0.0001$ \\
LVEF (\%) & $62.5 \pm 7.0$ & & \\
\hline
\end{tabular}

Note: $\mathrm{LVEF}=$ Left ventricular ejection fraction, NS $=$ not significant.

Table 2. Comparison of pre and post contrast-enhanced myocardial and left ventricular lumen $\mathrm{T} 1$ values between control and DCM groups.

\begin{tabular}{cccc}
\hline & Control & DCM & $P$ \\
\hline $\begin{array}{c}\text { Pre M T1 value } \\
\text { (msec) }\end{array}$ & $519.7 \pm 30.4$ & $581.3 \pm 58.3$ & 0.005 \\
$\begin{array}{c}\text { Post M T1 } \\
\text { value (msec) }\end{array}$ & $390.4 \pm 19.3$ & $344.5 \pm 31.6$ & $<0.0001$ \\
$\begin{array}{c}\text { Pre L T1 value } \\
\text { (msec) }\end{array}$ & $731.4 \pm 64.4$ & $741.2 \pm 92.5$ & NS \\
$\begin{array}{c}\text { Post L T1 value } \\
\text { (msec) }\end{array}$ & $299.3 \pm 35.5$ & $257.5 \pm 46.1$ & 0.002 \\
\hline
\end{tabular}

Note: NS = not significant.

Table 3. Comparison of four quantification methods of T1 value between control and DCM groups.

\begin{tabular}{cccc}
\hline & Control & DCM & $P$ \\
\hline Post M T1 value (msec) & $390.4 \pm 19.3$ & $344.5 \pm 31.6$ & $<0.0001$ \\
$\begin{array}{c}\text { Pre M T1 value - post M } \\
\text { TI value (msec) }\end{array}$ & $134.0 \pm 28.9$ & $239.9 \pm 64.2$ & $<0.0001$ \\
$\begin{array}{c}\text { Post M T1 value/post L } \\
\text { T1 value }\end{array}$ & $1.31 \pm 0.13$ & $1.37 \pm 0.21$ & 0.3422 \\
$\begin{array}{c}\text { (pre M T1 value - post } \\
\text { M T1 value)/(pre L T1 }\end{array}$ & $0.30 \pm 0.60$ & $0.50 \pm 0.11$ & $<0.0001$ \\
value - post L T1 value) & & & \\
\hline
\end{tabular}

Note: $\mathrm{M}=$ myocardial, $\mathrm{L}=$ left ventricular lumen, $\mathrm{DCM}=$ dilated cardiomyopathy.

groups in post M T1 value/post T1 value.

In the DCM groups, (pre M T1 value-post M T1 value)/(pre $L$ T1 value-post $L$ T1 value) was the most significantly related to the left ventricular ejection fraction $(r=0.66 ; P<0.0001)$ (Figure 2). The other quantification methods (post M T1 value, pre M T1 value-post M T1 value, and post M T1 value/post $L$ T1 value) had smaller positive correlations than (pre M T1 value-post M T1 value)/(pre $L$ T1 value-post $L$ T1 value) with the left ventricular ejection fraction (post $\mathrm{M}$ T1 value; $r=$ 0.53; $P<0.0001$ ) (Figure 3), pre M T1 value-post M T1 value $(r=-0.47 ; P<0.0001)$ (Figure 4), and post M T1 value/post $\mathrm{L}$ T1 value $(r=0.10 ; P=0.43)$ (Figure 5).

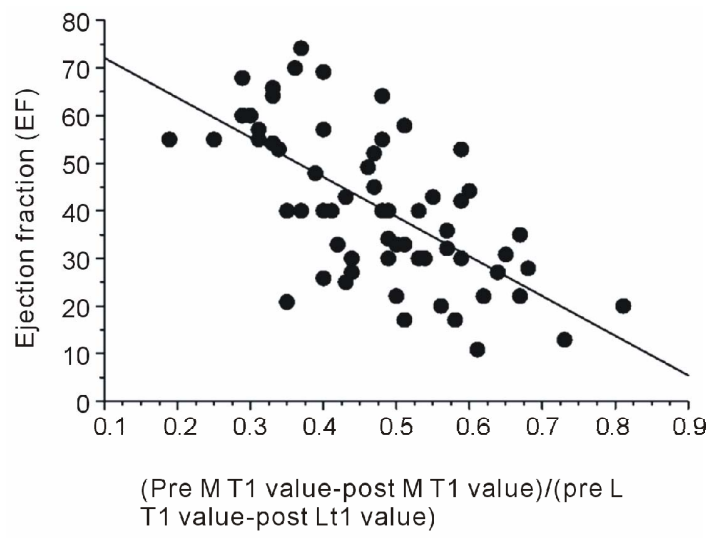

Figure 2. Scatterplot shows correlation between left ventricular ejection fraction and (pre M T1 value-post M T1 value)/(pre $L$ T1 value-post $L$ T1 value) in patients with DCM. (Pre M T1 value-post $M T 1$ value)/(pre $L T 1$ value-post $L T 1$ value) was most significantly related to left ventricular ejection fraction $(r=0.66 ; P<0.0001)$.

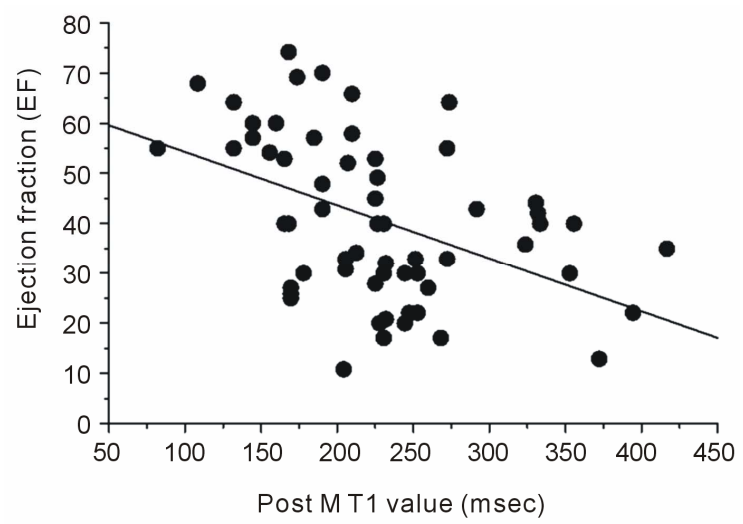

Figure 3. Scatterplot shows correlation between left ventricular ejection fraction and post $M T 1$ value in patients with DCM. Post M T1value was significantly related to left ventricular ejection fraction $(r=0.53 ; P<0.0001)$.

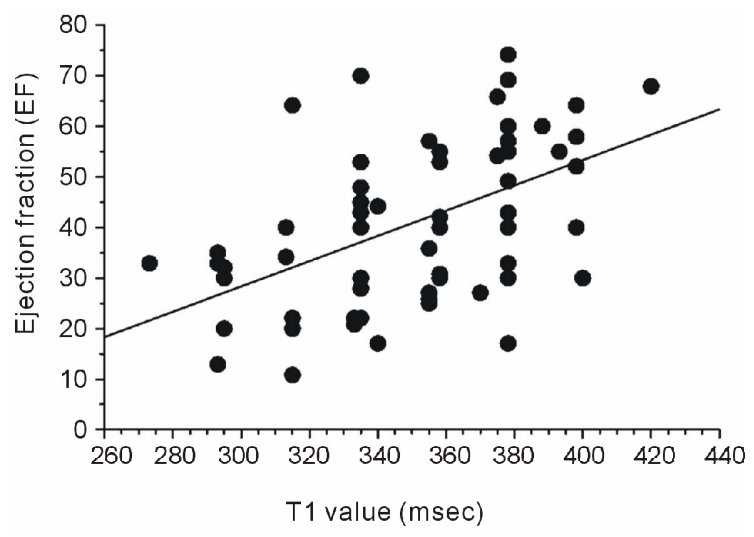

Figure 4. Scatterplot shows correlation between left ventricular ejection fraction and pre $M T 1$ value-post $M T 1$ value in patients with DCM. Pre M T1 value-post M T1 value was significantly related to left ventricular ejection fraction $(r=-0.47 ; P<0.0001)$. 


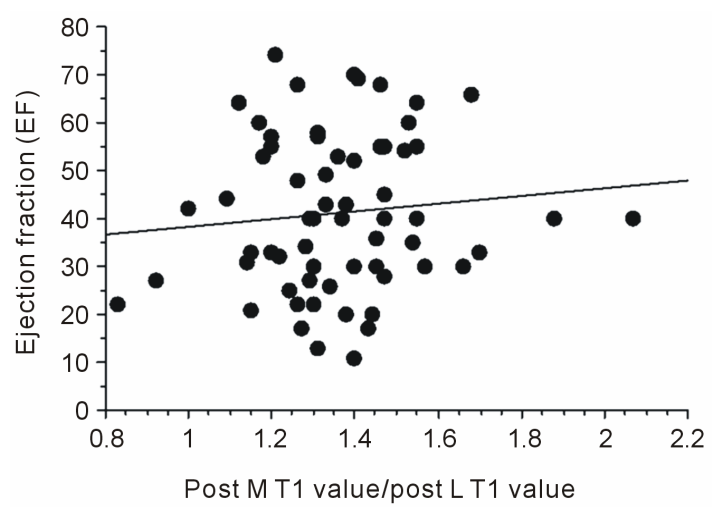

Figure 5. Scatterplot shows correlation between left ventricular ejection fraction and post $M T 1$ value/post $L$ T1 value in patients with DCM. Post M T1 value/post $L$ T1 value was not significantly related to left ventricular ejection fraction $(r=0.10 ; P=0.43)$.

\section{Discussion}

In patients with DCM, an important mechanism for the occurrence of arrhythmias and failure to respond to treatment is the presence of myocardial fibrosis [10-13]. Therefore, in patients with DCM, fibrosis is one of the most important risk factors for mortality.

Delayed enhanced MRI has enabled depiction of myocardial damage with high spatial resolution in various myocardial diseases. Delayed enhanced cardiac MRI has been used to evaluate focal myocardial fibrosis, but it is difficult to evaluate diffuse fibrosis of the myocardium. Prior studies have identified pathologic findings of myocardial fibrosis and disarray can show enhancement on delayed enhanced images, but delayed enhancement related to disarray is usually faint. Measurement of contrast-enhanced myocardial $\mathrm{T} 1$ value can provide an estimate of diffuse fibrosis of the myocardium $[1,5,7]$. Therefore, the prior studies used contrast-enhanced myocardial T1 value, which is easily calculated on a workstation [14-16]. In addition, T1 value was used as the optimal inversion time when delayed enhanced MR images were acquired.

According to previous study, the T1 values were 273 420 msec, 15 minutes after administration of contrast material in patients with DCM [5]. This quantification method has been postulated to be useful as this contrastenhanced myocardial $\mathrm{T} 1$ value was significantly shorter in the patient group than in the control group. However, this quantification method does not consider the influence of the contrast medium of the blood pool and thus this quantification method might not enable precise determination of the enhancement of myocardium. Another prior study identified quantification methods such as post M T1 value/post L T1 value, which considered the influence of the contrast medium of the blood pool [17], but this quantification method did not measure the dif- ference between post M T1 value and pre M T1 value, or between post $\mathrm{L} \mathrm{T} 1$ value and pre $\mathrm{L} \mathrm{T} 1$ value. For these reasons, this quantification method might not evaluate the enhancement of myocardium precisely.

To our knowledge, this is the first study to determine pre and post contrast-enhanced myocardial and left ventricular lumen T1 values. This study compared several quantification methods.

In this study, post M T1 value, pre M T1 value-post $M$ T1 value, and (pre M T1 value-post M T1 value)/(pre L T1 value-post $L T 1$ value) showed statistically significant differences between patient and control groups. These methods could be useful diagnostic tools for DCM. However, because the quantification method of post M T1 value did not measure a difference between post M T1 value and pre $\mathrm{M} T 1$ value, this quantification method may not evaluate the enhancement of myocardium precisely. Moreover, because this quantification method may not measure the difference between post $\mathrm{L}$ T1 value and pre $\mathrm{L} T 1$ value, this quantification method did not consider the influence of the contrast medium of the blood pool.

The quantification method of pre M T1 value-post M $\mathrm{T} 1$ value may not measure the difference between post $\mathrm{L}$ $\mathrm{T} 1$ value and pre $\mathrm{L} T 1$ value. Because this quantification method did not consider the influence of the contrast medium of the blood pool, this quantification method may not evaluate the enhancement of myocardium precisely.

The quantification method of post M T1 value/post L T1 value did not show a significant difference between the patient and control groups, although this quantification method considers the influence of the contrast medium of the blood pool.

The quantification method of (pre M T1 value-post $M$ $\mathrm{T} 1$ value)/(pre $\mathrm{L} T 1$ value-post $\mathrm{L}$ T1 value) measured the difference between post $\mathrm{M} T 1$ value and pre $\mathrm{M} T 1$ value, and between post $\mathrm{L} \mathrm{T} 1$ value and pre $\mathrm{L} \mathrm{T} 1$ value. Because this quantification method considers the influence of the contrast medium of the blood pool, this method may evaluate the enhancement of myocardium most precisely among the four methods.

In prior studies, left ventricular ejection fraction was suggested to be associated with serious clinical symptoms [18-20]. According to our results, (pre M T1 value-post $M T 1$ value)/(pre $L$ T1 value-post $L$ T1 value) was the most significantly related to the left ventricular ejection fraction $(r=0.66 ; P<0.0001)$ in the DCM group. Our study shows that the quantification method of (pre M T1 value-post M T1 value)/(pre L T1 value-post L T1 value) considers the influence of the contrast medium of the blood pool and evaluates the enhancement of myocardium. Therefore, this method may be the most reliable diagnostic method to evaluate the extent of myo- 
cardial fibrosis non-invasively.

There were several limitations to this study. First, we used inversion times to measure a single slice. Ideally, measurement of the whole myocardium is needed to evaluate diffuse fibrosis. Therefore, further studies regarding markers of fibrosis are needed. In addition, it is not easy to draw a myocardial boundary. This process may potentially bias the results.

Second, this study lacked a comparison of severity of myocardial fibrosis, histologically. However, it may be impossible to correlate the areas of fibrosis on biopsy with the areas seen on MRI.

Third, we evaluated only four quantification methods and we must carry out further examination to find a more useful quantification method.

\section{Conclusion}

In conclusion, (pre M T1 value-post M T1 value)/(pre L $\mathrm{T} 1$ value-post $\mathrm{L} \mathrm{T} 1$ value), which considered the influence of the contrast medium of the blood pool, evaluated the enhancement of myocardium precisely. This method was the most significantly related to the left ventricular ejection fraction. These data suggest that (pre M T1 value-post $\mathrm{M} \mathrm{T}$ 1 value)/(pre $\mathrm{L}$ T1 value-post $\mathrm{L} \mathrm{T} 1$ value) is the most reliable quantification method to estimate the severity of DCM.

\section{REFERENCES}

[1] L. Iles, H. Pfluger, A. Phrommintikul, J. Cherayath, P. Aksit, S. N. Gupta, D. M. Kaye and A. J. Taylor, "Evaluation of Diffuse Myocardial Fibrosis in Heart Failure with Cardiac Magnetic Resonance Contrast-Enhanced T1 Mapping," Journal of the American College of Cardiology, Vol. 52, No. 19, 2008, pp. 1574-1580.

doi:10.1016/j.jacc.2008.06.049

[2] T. M. Koelling, R. S. Chen, R. N. Lubwama, G. J. L'Italien and K. A. Eagle, "The Expanding National Burden of Heart Failure in the United States: The Influence of Heart Failure in Women," American Heart Journal, Vol. 147, No. 1, 2004, pp. 74-78. doi:10.1016/j.ahj.2003.07.021

[3] D. L. Mann, "Mechanisms and Models in Heart Failure: A Combinatorial Approach," Circulation, Vol. 100, 1999, pp. 999-1008. doi:10.1161/01.CIR.100.9.999

[4] T. F. Ismail, S. K. Prasad and D. J. Pennell, "Prognostic Importance of Late Gadolinium Enhancement Cardiovascular Magnetic Resonance in Cardiomyopathy," Heart, Vol. 98, No. 6, 2012, pp. 438-442. doi:10.1136/heartinl-2011-300814

[5] E. Sueyoshi, I. Sakamoto and M. Uetani, "Contrast-Enhanced Myocardial Inversion Time at the Null Point for Detection of Left Ventricular Myocardial Fibrosis in Patients with Dilated and Hypertrophic Cardiomyopathy: A Pilot Study," American Journal of Roentgenology, Vol. 194, No. 4, 2010, pp. W293-W298.

\section{doi:10.2214/AJR.09.3414}

[6] P. Richardson, W. McKenna, M. Bristow, B. Maisch, B. Mautner, J. O'Connell, E. Olsen, G. Thiene, J. Goodwin, I. Gyarfas, I. Martin and P. Nordet, "Report of the 1995 World Health Organization/International Society and Federation of Cardiology Task Force on the Definition and Classification of Cardiomyopathies," Circulation, Vol. 93, 1996, pp. 841-842. doi:10.1161/01.CIR.93.5.841

[7] P. Sparrow, D. R. Messroghli, S. Reid, J. P. Ridgway, G. Bainbridge and M. U. Sivananthan, "Myocardial T1 Mapping for Detection of Left Ventricular Myocardial Fibrosis in Chronic Aortic Regurgitation: Pilot Study," American Journal of Roentgenology, Vol. 187, No. 6, 2006, pp. W630-W635. doi:10.2214/AJR.05.1264

[8] D. R. Messroghli, S. Plein, D. M. Higgins, K. Walters, T. R. Jones and J. P. Ridgway, "Human Myocardium: Single-Breath-Hold MR T1 Mapping with High Spatial Resolution-Reproducibility Study," Radiology, Vol. 238, No. 3, 2006, pp. 1004-1012. doi:10.1148/radiol.2382041903

[9] K. H. Zou, K. Tuncali and S. G. Silverman, "Correlation and Simple Linear Regression," Radiology, Vol. 227, No. 3, 2003, pp. 617-622. doi:10.1148/radiol.2273011499

[10] R. J. Kim, E. Wu, A. Rafael, E. L. Chen, M. A. Parker, O. Simonetti, F. J. Klocke, R. O. Bonow and R. M. Judd, "The Use of Contrast-Enhanced Magnetic Resonance Imaging to Identify Reversible Myocardial Dysfunction," The New England Journal of Medicine, Vol. 343, No. 20, 2000, pp. 1445-1453. doi:10.1056/NEJM200011163432003

[11] R. G. Assomull, S. K. Prasad, J. Lyne, G. Smith, E. D. Burman, M. Khan and M. N. Sheppard, P. A. PooleWilson and D. J. Pennell, "Cardiovascular Magnetic Resonance, Fibrosis, and Prognosiin Dilated Cardiomyopathy," Journal of the American College of Cardiology, Vol. 48, No. 10, 2006, pp. 1977-1985. doi:10.1016/j.jacc.2006.07.049

[12] D. Bello, D. S. Fieno, R. J. Kim, F. S. Pereles, R. Passman, G. Song, A. H. Kadish and J. J. Goldberger, "Infarct Morphology Identifies Patients with Substrate for Sustained Ventricular Tachycardia," Journal of the American College of Cardiology, Vol. 45, No. 7, 2005, pp. 11041108. doi:10.1016/j.jacc.2004.12.057

[13] D. Bello, D. J. Shah, G. M. Farah, S. Di Luzio, M. Parker, M. R. Johnson, W. G. Cotts, F. J. Klocke, R. O. Bonow, R. M. Judd, M. Gheorghiade and R. J. Kim, "Gadolinium Cardiovascular Magnetic Resonance Predicts Reversible Myocardial Dysfunction and Remodeling in Patients with Heart Failure Undergoing Betablocker Therapy," Circulation, Vol. 108, 2003, pp. 1945-1953. doi:10.1161/01.CIR.0000095029.57483.60

[14] A. M. Huber, S. O. Schoenberg, C. Hayes, B. Spannagl, M. G. Engelmann, W. M. Franz and M. F. Reiser, "Phasesensitive Inversion-Recovery MR Imaging in the Detection of Myocardial Infarction," Radiology, Vol. 237, No. 3, 2005, pp. 854-860. doi:10.1148/radiol.2373041483

[15] J. N. Oshinski, Z. Yang, J. R. Jones, J. F. Mata and B. A. French, "Imaging Time after Gd-DTPA Injection is Critical in Using Delayed Enhancement to Determine Infarct 
Size Accurately with Magnetic Resonance Imaging," Circulation, Vol. 104, 2001, pp. 2838-2842.

doi:10.1161/hc4801.100351

[16] C. Klein, S. G. Nekolla, F. M. Bengel, M. Momose, A. Sammer, F. Haas, B. Schnackenburg, W. Delius, H. Mudra, D. Wolfram and M. Schwaiger, "Assessment of Myocardial Viability with Contrast Enhanced Magnetic Resonance Imaging: Comparison with Positron Emission Tomography," Circulation, Vol. 105, 2002, pp. 62-167. doi:10.1161/hc0202.102123

[17] A. K. Kono, N. Yamada, M. Higashi, S. Kanzaki, H. Hashimura, Y. Morita, T. Sakuma, T. Noguchi, H. Naito and K. Sugimura, "Dynamic Late Gadolinium Enhancement Simply Quantified Using Myocardium to Lumen Signal Ratio: Normal Range of Ratio and Diffuse Abnormal Enhancement of Cardiac Amyloidosis," Journal of Magnetic Resonance Imaging, Vol. 34, No. 1, 2011, pp. 50-55. doi:10.1002/jmri.22602
[18] J. J. Bax, D. Poldermans, A. Elhendy, J. H. Cornel, E. Boersma, R. Rambaldi, J. R. Roelandt and P. M. Fioretti, "Improvement of Left Ventricular Ejection Fraction, Heart Failure Symptoms and Prognosis after Revascularization in Patients with Chronic Coronary Artery Disease and Viable Myocardium Detected by Dobutamine Stress Echocardiography," Journal of the American College of Cardiology, Vol. 34, No. 1, 1999, pp. 163-169. doi:10.1016/S0735-1097(99)00157-6

[19] F. Cacciapuoti, "Are Clinical Heart Failure and Ejection Fraction Always Connected?" Open Heart Failure Journal, Vol. 3, 2010, pp. 1-8.

[20] I. Nishi, T. Noguchi, Y. Iwanaga, S. Furuichi, N. Aihara, H. Takaki and Y. Goto, "Effects of Exercise Training in Patients with Chronic Heart Failure and Advanced Left Ventricular Systolic Dysfunction Receiving $\beta$-Blockers," Circulation Journal, Vol. 75, No. 7, 2011, pp. 1649-1655. doi:10.1253/circj.CJ-10-0899 